\title{
Sincinesia oral-ocular: una complicación de la parálisis facial periférica Reporte de un caso
}

\author{
Arriaga-Rivera Javier*, Ramírez-Cervantes José de Jesús**, Alejo-González Mónica Patricia***
}

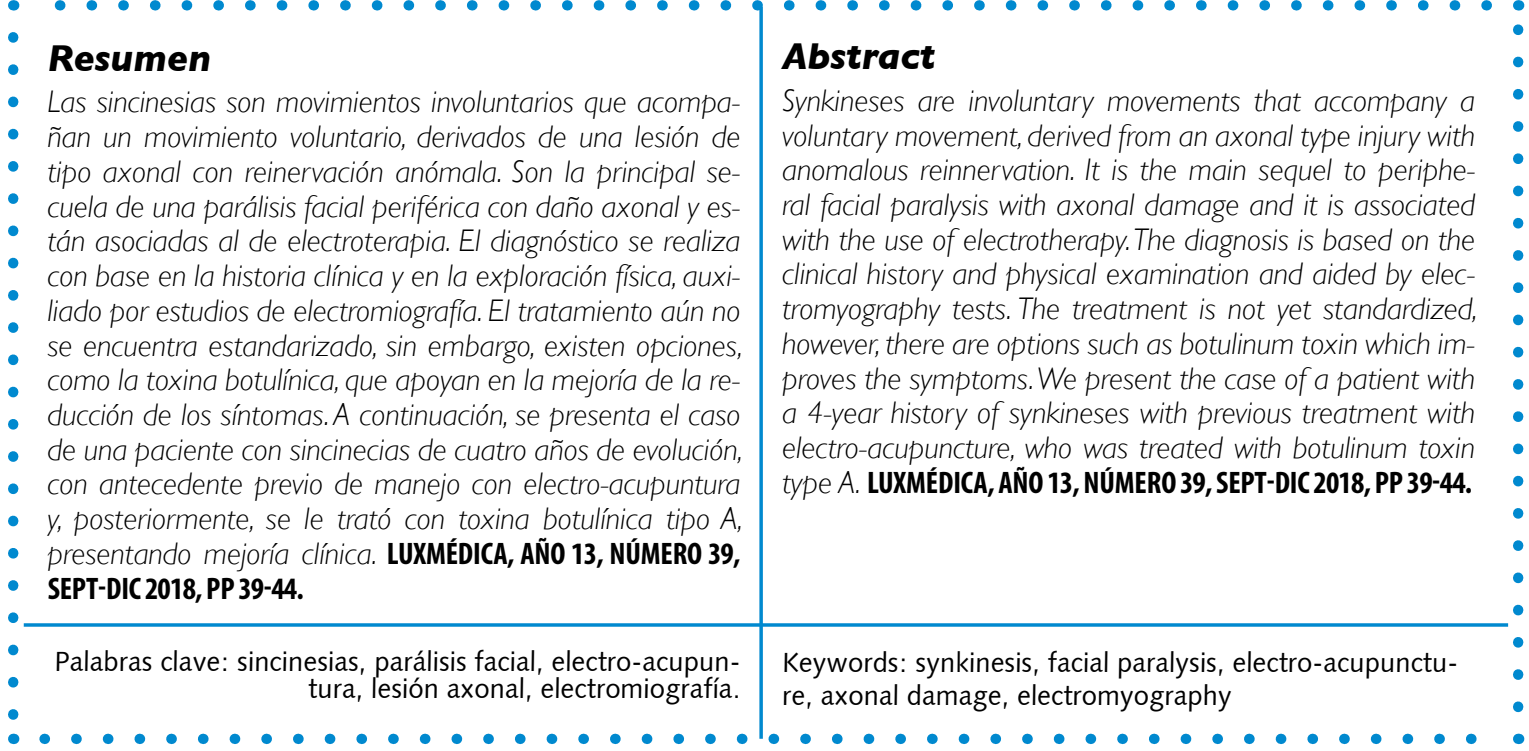

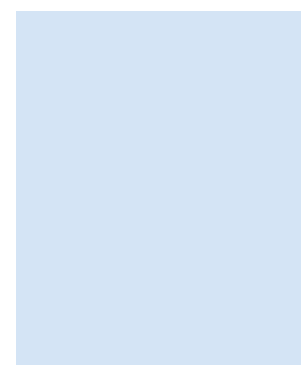

\section{Introducción}

La parálisis facial periférica (PFP) tiene una incidencia de 20-30 casos por cada 100000 habitantes. El 70\% de los casos presentan una recuperación completa en un periodo de tres meses (para los casos desmielinizantes).1-2 Sin embargo, aquellos con una recuperación incompleta presentarán debilidad, contracturas, atrofia, sincinesias o mioquimias, que afectan la calidad de vida y estado de ánimo del individuo debido al impacto psicosocial. ${ }^{3,4}$

\footnotetext{
* Médico especialista en Rehabilitación

** Medico pasante de servicio social. Universidad Cuauhtémoc

*** Médico especialista en Rehabilitación y Electrodiagnóstico
}

Fecha de recibido: 10 abril 2018

Fecha de aceptación: 10 junio 2018

Correspondencia: Dr Javier Arriaga Rivera. DIF Estatal Aguascalientes, Centro de Rehabilitación e Inclusión Social (CRIS). Av. Mahatma Gandhi SN, esq. República de Paraguay, colonia Agricultura Código Postal 20234. Aguascalientes, Ags., México. Teléfono 0144991760 00. Correo electrónico: movimedic-ags@hotmail.com 
La sincinecia es definida como un movimiento involuntario que acompaña un movimiento voluntario asociada a una lesión de tipo axonal ${ }^{1,5}$ y provoca una reinervación anómala, alteración de los patrones de activación muscular y que suele incrementar el riesgo de aparición al aplicar tratamiento basados en electroterapia. ${ }^{4,5}$

Los tipos de sincinesia más comunes son: oral-ocular, que se manifiesta con el cierre involuntario del ojo durante el movimiento voluntario de la boca, y la sincinesia ocular-oral, en donde se observa el movimiento involuntario de la boca durante el cierre voluntario del ojo. Aparecen con mayor frecuencia entre los tres y los cuatro meses después de la lesión nerviosa y, en algunos casos, dos años después de una lesión con recuperación incompleta. ${ }^{1,6}$

En el Centro de Rehabilitación e Inclusión Social de Aguascalientes (CRIS), la PFP representa el $2.7 \%$ de los motivos de consulta, el mayor número de casos se concentran en el grupo de edad de 12-34 años, y 35-59 años, con mayor frecuencia en mujeres. Las secuelas reportadas en este CRIS son raras; sin embargo, los pacientes con una lesión axonal, o en la porción intrapetrosa del nervio facial, tienen un mayor riesgo de desarrollar sincinecias.

A continuación presentamos el caso de una mujer de 48 años que acudió a consulta por presentar mioquimias y sincinecias del músculo orbicular izquierdo, asociada a movimientos orales de cuatro años de evolución con antecedente de parálisis facial tratada con electro-acupuntura.

\section{Presentación del caso clínico}

Se presenta el caso de una paciente femenina de 48 años de edad con antecedentes de hipertensión arterial controlada con losartan (50 mg cada 24 horas), intolerancia a la glucosa en ayunas con manejo a base de metformina $850 \mathrm{mg}$ cada 24 horas y trastorno de ansiedad, bajo seguimiento por médico, con clonazepam $2.5 \mathrm{mg} / \mathrm{ml}$ (5 gotas cada 24 horas).

Inició su padecimiento hace cuatro años con presencia de debilidad en musculatura facial izquierda en forma súbita y disacusia sin un factor etiológico identificado, por lo que acudió a valoración con técnico auxiliar de la salud; éste realiza manejo a base de electro-acupuntura por diez sesiones, y se presenta mejoría de la movilidad en músculos faciales; sin embargo, cuatro meses después, identifica un cierre involuntario del ojo izquierdo y mioquimia del orbicular ipsilateral. Fue valorada por un médico, quien establece el diagnóstico de parálisis facial por estrés e iniciando manejo con clonazepam, sin mejoría de los síntomas. La paciente manifestó el incremento en el cierre ocular asociado al habla, por lo cual se le solicitó un estudio de tomografía de cráneo, con reporte normal. Al no encontrar otros datos neurológicos, se solicitó una valoración psicológica con probable diagnóstico de síndrome psicosomático, y se canaliza a rehabilitación para valoración. En octubre de 2017, es valorada por medicina de rehabilitación con diagnóstico de envío de parálisis facial, mostrando una exploración neurológica normal con debilidad para el orbicular del ojo izquierdo 
en escala 2/3 de Lovett. Durante el interrogatorio, se apreció una sincinecia del tipo oral-ocular izquierda de puntuación 3 en escala de movimientos asociados de Zander Olsen y 71 puntos en (SAQ) Sinkynesis Assesment Questionarie (figura 1), (tablas 1,2). Por tal motivo, se realizó estudio de electromiografía (EMG) del nervio facial para documentar lesión nerviosa y descartar alteraciones de la placa neuromuscular con prueba de estimulación repetitiva del orbicular del ojo, y se identificó una lesión de tipo axonal para la rama terminal del orbicular del ojo (figura 2); las pruebas de estimulación repetitiva fueron normales. El patrón por estudio de aguja del músculo orbicular del ojo reportó un reclutamiento disminuido del potencial de acción de unidad motora, sin datos de re-inervación. Finalmente, se procedió a realizar tratamiento de rehabilitación con reeducación muscular frente al espejo y aplicación de toxina botulínica (TB) 25 UI en orbicular izquierdo y 15 UI en el risorio izquierdo. Se presentó una disminución de las sincinesias y mioquimias, con una puntuación de 1 en escala de movimientos asociados de Zander Olsen y 46 puntos en (SAQ) Sinkynesis Assesment Questionarie (ver figura 1B).

\section{Discusión}

La sincinesia es la secuela más común reportada entre el $9 \%$ y el $55 \%$ de los casos debido a una lesión en la porción intrapetrosa del nervio facial y/o del tipo axonal. El mecanismo fisiopatológico no está del todo claro, sin embargo, se han propuesto las siguientes teorías: a. acoplamiento efáptico de impulsos entre los axones; $b$. regeneración aberrante de las fibras del nervio facial; c. reorganización sináptica dentro del núcleo del nervio facial. ${ }^{8} \mathrm{El}$ diagnóstico es eminentemente clínico, basado en la historia clínica y en la exploración física. Los auxiliares para el mismo son la EMG del nervio facial, mientras que el diagnóstico diferencial debe realizarse con distrofia fascio escapulo humeral y miastenia gravis ocular.

El estudio de EMG tiene un valor diagnóstico y pronóstico relevante para las PFP que no presenten recuperación en los primero 8 días o que cuenten con una clasificación de House-Brackmann IV-VI, lo cual permite tanto identificar los casos con lesión axonal, como evaluar la evolución del paciente. ${ }^{7,20}$ En general, todas las lesiones de nervio periférico, incluyendo las del nervio facial, deben correlacionarse con base en los criterios de Seddon y Sunderland (figura 3), lo cual permite conocer el tipo, el grado, el comportamiento, y el pronóstico de la lesión .18, 19

En un estudio realizado por Balager con una población promedio de 47 años, el $31.9 \%$ de los pacientes con PFP presentó alguna secuela y, de éstos, el $24 \%$ fueron mioquimias o sincinesias con un tiempo de aparición que variaba entre los 3 a 24 meses; predominaban mujeres con una lesión axonal mayor del 50\%, documentada por EMG. ${ }^{7}$ En comparación con estos datos, en el CRIS, ubicado en la ciudad de Aguascalientes, se han tratado 20 casos de parálisis facial periférica, entre octubre de 2017 y marzo de 2018. La terapia ha consistido en ejercicios frente al espejo, de los cuales sólo un caso (tratado previamente con electro-acupuntura) presentó secuelas, con inicio de sincinecias cuatro meses después del cuadro de PFP, por lo que se realizó EMG del nervio facial y prueba de estimulación repetitiva. Se concluyó que se trataba de una lesión axonal del $69 \%$, y se descartaron enfermedades de placa neuromuscular, lo cual ha sido reportado en otras publicaciones. 7,8 


\section{Tratamiento}

Actualmente, el tratamiento de la PFP ha sido bien documentado mediante metanálisis y guías de práctica clínica, y se ha coincidido en que el uso de esteroides en las primeras 72 horas y la reeducación muscular frente al espejo son las opciones con mejor evidencia científica.9,10, 20 Los recursos como la acupuntura, el láser y el ultrasonido no muestran ventaja en comparación con los esteroides y el ejercicio, por lo que su uso no es recomendable. ${ }^{9,11}$ Además, en relación con la electroterapia, se ha documentado un incremento del tono y tetania, así como efectos negativos que predisponen a sincinesias.9,16 Fargher también documentó una alteración del crecimiento axonal y de los mecanismos de reparación nerviosa asociados al uso de electroterapia, y las revisiones sistemáticas concluyen que no existen beneficios del uso de electroterapia contra el ejercicio terapéutico y esteroides, por lo que esta práctica ha caído en desuso..$^{9,15-17}$
En relación con el tratamiento de las complicaciones, como las sincinesias y las mioquimias, existe un limitado número de estudios y no hay un consenso de su abordaje. Algunos reportes de casos emplean la TB desde la década de los noventa y la reeducación muscular guiada con electromiografía como opciones de tratamiento. ${ }^{12-14}$ Filipo evaluó la respuesta de las sincinesias al tratamiento con TB en 41 pacientes, y se mostraba una diferencia estadística significativa a favor del grupo de tratamiento; esto coincide con lo reportado por Roggenkamper, el cual utilizó dicho tratamiento en 23 pacientes, con mejoría clíni$c a$, y en el caso de Wiener, se expone que la TB es efectiva y fácil de utilizar, infiltrando 1-5 UI (Botox) en diferentes músculos. Para el caso expuesto en este articulo, se utilizaron 25 UI de TB (Xeomin) en el orbicular del ojo izquierdo, y $15 \mathrm{UI}$ en el risorio ipsilateral, lo cual ayudó a la mejoría en relación a la escala de movimientos asociados de Zander Olsen y (SAQ) Sinkynesis Assesment Questionarie posterior a 2 semanas de la infiltración.

\section{Conclusiones}

La parálisis facial periférica es un padecimiento relativamente común y en general, se asocia a un buen pronóstico; sin embargo, en los pacientes con daño axonal, la recuperación puede ser tardía o incompleta y producir secuelas como las sincinesias.

En el caso presentado, la paciente ya tenía cuatro años con una sincinesia, que afectaba su calidad de vida y ámbito psicosocial. Respecto a este caso, hay varios puntos sobresalientes. El primero fue la ausencia de tratamiento farmacológico establecido por las guías de práctica clínica. El segundo fue que la paciente acudió con un técnico auxiliar de salud, sin el aval de un médico especialista en rehabilitación, lo cual pone en duda el abordaje de electro-acupuntura que se otorgó. El tercero, que nunca se le solicitó una electromiografía que documentara la lesión axonal. Finalmente, por el largo tiempo de evolución, la paciente acudió con varios profesionales de la salud, sin tener claro su padecimiento. Todos los puntos anteriores retardaron el diagnóstico y abordaje.

Con la experiencia terapéutica en esta paciente, considerando que el objetivo es el control de los movimientos involuntarios, la TB fue la opción de tratamiento que demostró efectividad, mientras que las escalas de Zander Olsen y el (SAQ) Sinkynesis Assesment Questionarie son herramientas confiables para el seguimiento de estos pacientes. 

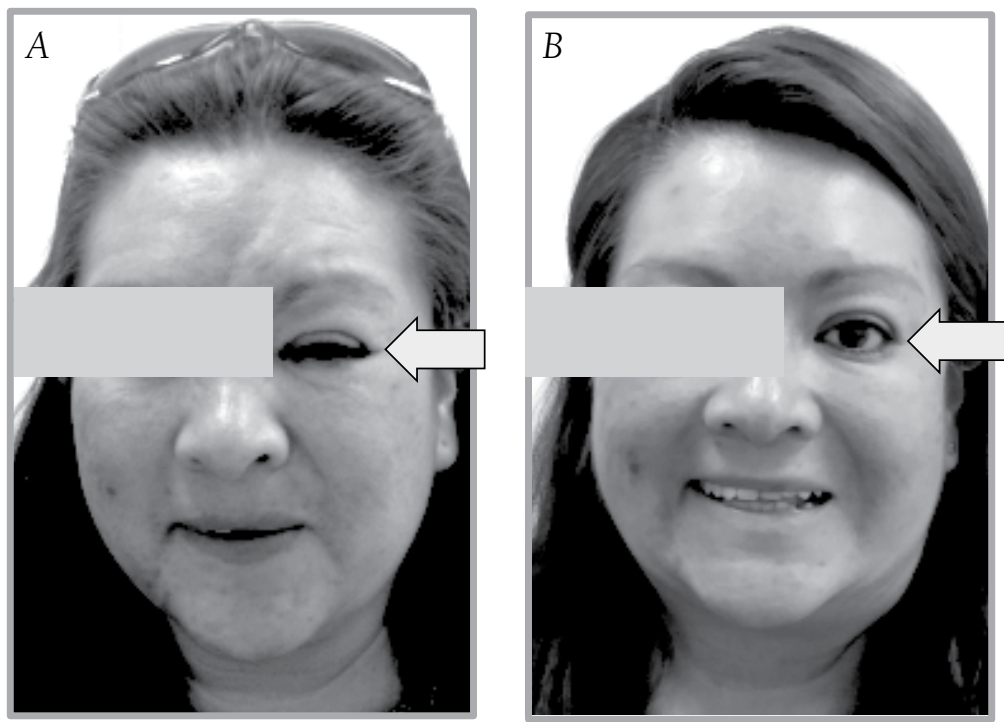

Figura 1.-A) Muestra sincinesia oral-ocular izquierda. B) Muestra mejoría del movimiento en orbicular 2 semanas posterior a la aplicación de TB.

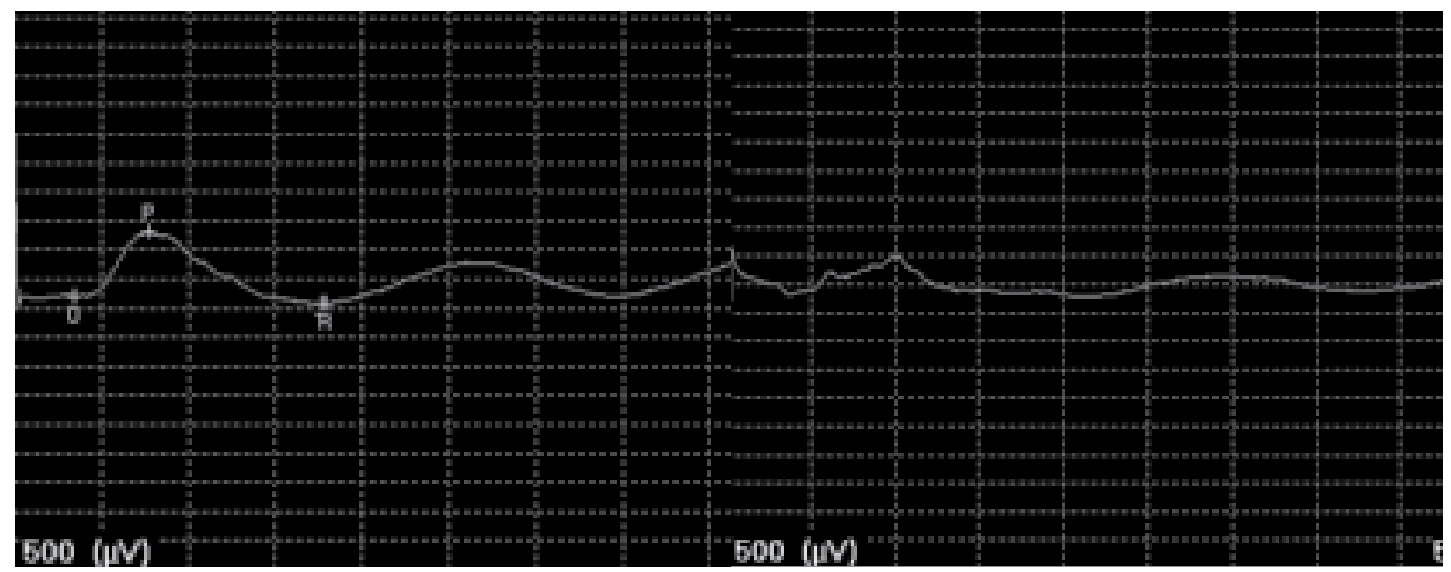

Figura 2.- A) Muestra el PAMC del orbicular derecho. B) Muestra el PAMC del orbicular izquierdo con morfología alterada y disminución de la amplitud.

\begin{tabular}{|lll}
\hline \multicolumn{2}{|c|}{ Estadio } & \multicolumn{1}{c}{ Anatomía patológica } \\
\hline Seddon & Sunderland & \\
\hline Neuropraxia & I & Desmielinización segmentaria \\
\hline Axonotmesis & II & $\begin{array}{l}\text { Axón lesionado pero con endoneuro intacto } \\
\text { (circunstancias óptimas para regeneración) }\end{array}$ \\
\hline Axonotmesis & III & $\begin{array}{l}\text { Discontinuidad de axón, discontinuidad del tubo endoneural, con } \\
\text { preservación del perineuro }\end{array}$ \\
\hline Axonotmesis & IV & $\begin{array}{l}\text { Pérdida de continuidad de axones, endoneuro, perineurio y fascícu } \\
\text { los; epineuro intacto (neuroma en continuidad) }\end{array}$ \\
\hline Neurotmesis & $\mathrm{V}$ & Pérdida de continuidad de todo el tronco nervioso \\
\hline
\end{tabular}

Figura 3.- Clasificación de lesión de nervio periférico Seddon y Sunderland. ${ }^{19}$ 


\section{Tabla I}

\section{Movimientos asociados de Zander Olsen. ${ }^{21}$}

\begin{tabular}{|ll|}
\hline \multicolumn{1}{|c|}{ Movimientos asociados (sincinesias) } \\
\hline 0 & Normal (no hay sincinesias) \\
\hline 1 & Sincinesias mínimas durante la contracción voluntaria vigorosa \\
\hline 2 & Sincinesias claras durante los movimientos espontáneos, parpadeo, sonrisa, etc. \\
\hline 3 & Sincinesia marcada en todos los movimientos \\
\hline
\end{tabular}

\section{Tabla 2}

(SAQ) Sinkynesis Assesment Questionarie. 22

\begin{tabular}{|ll|}
\hline \multicolumn{1}{|c|}{ Pregunta } \\
\hline 1 & Cuando sonrío, ¿mi ojo se cierra? \\
\hline 2 & Cuando hablo, ¿mi ojo se cierra? \\
\hline 3 & Cuando silbo o frunzo mis labios, ¿mi ojo se cierra? \\
\hline 4 & Cuando sonrío, ¿mi cuello se contrae o se tensa? \\
\hline 5 & Cuando cierro mis ojos, ¿mi cara se contrae o se tensa? \\
\hline 6 & Cuando cierro mis ojos, ¿mi comisura labial se mueve? \\
\hline 7 & Cuando cierro mis ojos, ¿mi cuello se contrae o se tensa? \\
\hline 8 & Cuando como, ¿mi ojo lagrimea? \\
\hline 9 & Cuando muevo mi cara, ¿mi barbilla desarrolla un área con hoyuelo? \\
\hline
\end{tabular}

1.- rara vez o nada. 2.- ocasionalmente. 3.- a veces o levemente. 4.- la mayor parte del tiempo o moderadamente 5.- todo el tiempo o severamente. Suma de items 1-9/45 ×100.

\section{Bibliografía}

1.- Rodríguez. et al. Parálisis facial periférica. Tratamientos y consideraciones. Arch Neurocien. 2011;16,3:148-155.

2.- Pourmomeny AA, Asadi S. Management of Synkinesis and Asymmetry in Facial Nerve Palsy: A Review Article. Iran J Otorhinolaryngol.2014;26 (4):251-256.

3.- Pereira LM. Facial exercise therapy for facial palsy: systematic review and meta-analysis. Clin Rehabil 2011; 25(25): 649-58.

4.- Pepper J-P. Selective chemodenervation with botulinum toxin in facial nerve disorders. Operative Techniques. Otolaryngol Head Neck Surg. 2012; 23(4): 297-305.

5.- Terzis JK KD. Therapeutic strategies in post-facial paralysis synkinesis in pediatric patients. J Plas Reconstruct Aesthet Surg 2012;68(8):1009-1018

6.- Nakamura K, Toda N, Sakamaki K, Kashima K, Takeda N. Biofeedback rehabilitation for prevention of synkinesis after facial palsy. Otolaryngology - Head and Neck Surgery 2003; 128(4): 539-43.

7.- Balaguer GR.et al. Secuelas de las parálisis faciales periféricas. Acta Otorrinolaringol Esp. 2010;61(2):89-93

8.- Münevver Ç. Hulki F, Çetin V. The Development of Synkinesis after Facial Nerve Paralysis. Eur Neurol 2000;43:147151.

9.- Teixeira LJ, Valbuza JS, PradoGF. Physical therapy for Bell's palsy (idiopathic facial paralysis). CochraneDatabase of Systematic Reviews 2011, Issue 12. Art. No.: CD006283. DOI: 10.1002/14651858.CD006283.pub3.

10.-Madhok VB, Gagyor I, Daly F, Somasundara D, Sullivan M, Gammie F, Sullivan F. Corticosteroids for Bell's palsy (idiopathic facial paralysis). Cochrane Database of Systematic Reviews 2016, Issue 7. Art. No.: CD001942. DOI: 10.1002/14651858.CD001942.pub5.
11. Chen N, Zhou M, He L, Zhou D, Li N. Acupuncture for Bell's palsy. Cochrane Database of Systematic Reviews 2010, Issue 8. Art. No.: CD002914. DOI: 10.1002/14651858. CD002914.pub5

12. Rodríguez OM. et al. Parálisis facial periférica. Tratamientos y Consideraciones. Arch Neurocien.2011;16(3):148-155

13. Filipo, R, Spahiu, I, Covelli, E, Nicastri, M. Bertoli, G. Botulinum toxin in the treatment of facial synkinesis and hyperkinesis. The Laryngoscope. 2012: 122(2):266-70.

14. Wiener A, Touloei K, Glick BP. A Novel Long-term Therapy of Facial Synkinesis with Botulinum Neurotoxins Type A and Fillers. J Clin Aesthet Dermatol.2011;4(3):45-49.

15. Garanhani MR. et aL. Physical therapy in peripheral facial paralysis: retrospective study. Rev Bras Otorrinolaringol.2007;73(1):112-5.

16. Fargher $\mathrm{K}$, Coulson S. Effectiveness of electrical stimulation for rehabilitation of facial nerve paralysis, Phys Ther Rev.2017:22(3-4):169-176

17. Arnulfo RJ, Rivera J, Torres R, Holguin E, Molina R. Effectiveness of Electro-stimulation as a Treatment for Bell's Palsy: An Update Review. J Nov Physiother .2015:5(2);1-4.

18. Mannarelli $G$, et al. Electrophysiological measures in facial paresis and Paralysis. Oper tech otolaryngol.2012; 23: 236247.

19. Campbell W. Evaluation and management of peripheral nerve Injury. Clinical Neurophysiology.2008:119; $1951-$ 1965.

20. Guía de práctica clínica para el diagnostico y tratamiento de la parálisis de Bell (parálisis facial idiopática), México: Secretaria de Salud; 2009.

21. Zander OP, Prediction of recovery in bell's palsy, Acta Neurol Scand. 1975;61(52):1-119.

22. Mehta RP, Wernickrobinson M, Hadlock TA, Validation of the Synkinesis Assessment Questionnaire, Laryngoscope. 2007;117(5):923-926. 


\section{Síndrome de Prune Belly Reporte de un caso}

Arias-Ulloa Raúl*, Arroyo-Torres Jessica Alejandra**, Pedroza-Soto Francisco Alejandro**

\begin{tabular}{|c|c|}
\hline Resumen & Abstract \\
\hline $\begin{array}{l}\text { El síndrome de Prune-Belly, también llamado síndrome de } \\
\text { abdomen en ciruela pasa, es una patología con una inciden- } \\
\text { cia baja, que ocurre principalmente en varones, aunque tam- } \\
\text { bién puede darse en mujeres (forma parcial). Este síndrome } \\
\text { presenta una triada característica: ausencia de músculos } \\
\text { abdominales, malformaciones del tracto urinario y criptor- } \\
\text { quidia bilateral. Se presenta el caso de un recién nacido en el } \\
\text { estado de Aguascalientes, con malformaciones compatibles } \\
\text { con el síndrome; actualmente se encuentra bajo tratamiento. } \\
\text { LUXMÉDICA, AÑ0 13, NÚMER0 39, SEPT-DIC 2018, PP 45-51. }\end{array}$ & $\begin{array}{l}\text { The prune belly syndrome, also called prune belly abdomen } \\
\text { syndrome, is a pathology with a low prevalence, which oc- } \\
\text { curs mainly in males, although it can also occur in women } \\
\text { (partial form). This syndrome presents a characteristic triad: } \\
\text { absence of abdominal muscles, malformations of the urinary } \\
\text { tract and bilateral cryptorchidism. We present the case of a } \\
\text { newborn in the state of Aguascalientes, with malformations } \\
\text { compatible with the syndrome; the patient is currently un- } \\
\text { der treatment. LUXMÉDICA, AÑO 13, NÜMERO 39, SEPT-DIC 2018, } \\
\text { PP 45-51. }\end{array}$ \\
\hline $\begin{array}{r}\text { Palabras clave: Síndrome de Prune-Belly, ausencia de } \\
\text { músculos abdominales. }\end{array}$ & $\begin{array}{l}\text { Keywords: prune belly syndrome, absence of abdominal } \\
\text { muscles }\end{array}$ \\
\hline
\end{tabular}

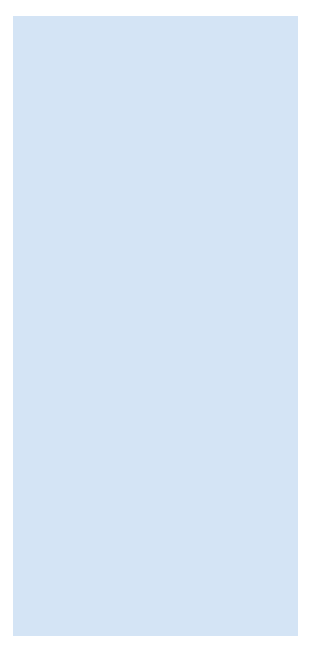

\section{Introducción}

El síndrome de Prune-Belly, también llamado síndrome de EagleBarret (síndrome del abdomen en ciruela pasa), consiste en el padecimiento, caracterizado por una triada clásica conformada por la ausencia de músculos abdominales, malformaciones del tracto urinario y criptorquidia bilateral. ${ }^{1}$

La incidencia en Estados Unidos es de 3.8 casos por cada 100,000 nacidos vivos, ${ }^{2}$ con predominio del sexo masculino sobre el femenino 20:1; sin embargo, en México no se han reportado datos epidemiológicos sobre el síndrome.

En 1839, Frölich describe por primera vez este síndrome en un niño que presentaba defecto de la musculatura abdominal, pecho en quilla y ausencia de descenso testicular. En 1895, Parker asoció el síndrome con malformaciones de las vías urinarias. En 1901,

\footnotetext{
Maestro en Salud Pública, profesor investigador de tiempo completo del Departamento de Salud Pública del Centro de Ciencias de la Salud de la Universidad Autónoma de Aguascalientes.
}

** Estudiantes del octavo semestre de la Carrera de Medicina del Centro de Ciencias de la Salud de la Universidad Autónoma de Aguascalientes.

Fecha de recibido 14 de mayo 2018

Fecha de aceptación: 14 de julio 2018

Correspondencia: MSP Raúl Arias Ulloa. Departamento de Salud Púbica. Edificio 107. Centro de Ciencias de la Salud. Campus Universitario. Universidad Autónoma de Aguascalientes. Av. Universidad 940. Ciudad Universitaria. Código postal 20131. Aguascalientes, Ags., México. Tel: (449) 9108434. Correo electrónico raul.arias@edu.uaa.mx 


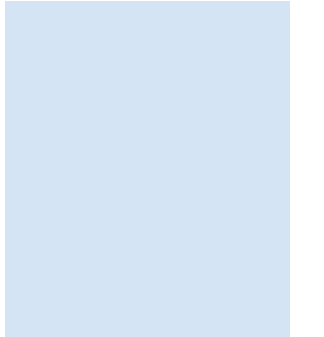

Osler lo nombró como "Prune-Belly" debido a la característica piel laxa sobre el abdomen, lo cual provoca el aspecto de ciruela pasa. En 1950, Eagle y Barret describieron 9 casos de pacientes con ausencia de musculatura abdominal, asociados a anormalidades genitourinarias.

Hasta el momento, la etiología del síndrome no ha sido descrita. Empero, se han sugerido varias teorías acerca del origen de éste.

1. Teoría del defecto mesodérmico: sugiere un defecto primario en la placa intermedia y lateral, lo cual provoca anomalías sobre la formación de los músculos abdominales, del mesonefros, los conductos paramesonéfricos y de los órganos del sistema urinario.

2. Teoría de la obstrucción uretral: ${ }^{3}$ propone que, secundario a ésta, existe una dilatación masiva de la vejiga, lo cual ocasiona un defecto sobre el desarrollo de los músculos abdominales, así como la imposibilidad del descenso testicular. El resultado de esto es una criptorquidia bilateral e hipoplasia de la pared abdominal.

Se ha intentado atribuir el síndrome a mutaciones genéticas. En un reporte de caso de una familia turca, se identificó una mutación en el gen CHRM3 localizado en el cromosoma 1q43. Dicho gen está involucrado en el desarrollo del epitelio renal y músculo vesical. ${ }^{4}$

Murray y cols. ${ }^{5}$ identificaron una deleción heterocigota completa en el gen HNF-1 $\beta$. Dicho factor se relaciona con la diferenciación endodérmica, que incluye el desarrollo de los derivados de los conductos mesonéfricos, los túbulos renales provenientes del metanefros y el desarrollo de la próstata.

El síndrome se caracteriza por una tríada clínica, conformada por la ausencia o hipoplasia de músculos abdominales, malformaciones del tracto urinario y criptorquidia bilateral.

La ausencia o hipoplasia de los músculos abdominales provoca el aspecto de piel laxa y arrugada de la pared abdominal muy semejante al aspecto de una ciruela pasa.

Dentro de las malformaciones del tracto urinario que se pueden presentar, se encuentran: displasia renal, reflujo vesicoureteral, dilatación de los ureteros, peristalsis ureteral inefectiva, dilatación vesical y persistencia de uraco. Se estima que hasta el $75 \%$ de los pacientes presentarán reflujo vesicoureteral. ${ }^{4}$ La displasia renal conllevará a una enfermedad renal crónica hasta en el $40 \%$ ó $50 \%$ de los pacientes, el cual requerirá, en algún momento de su vida, terapia de reemplazo renal. ${ }^{6}$

El síndrome puede ser clasificado según los criterios de Woodard (tabla 1): 


\section{Tabla I}

Movimientos asociados de Zander Olsen. ${ }^{21}$

Movimientos asociados (sincinesias)

\begin{tabular}{ll}
\hline 0 & Normal (no hay sincinesias) \\
\hline 1 & Sincinesias mínimas durante la contracción voluntaria vigorosa \\
\hline 2 & Sincinesias claras durante los movimientos espontáneos, parpadeo, sonrisa, etc. \\
\hline 3 & Sincinesia marcada en todos los movimientos \\
\hline
\end{tabular}

\section{Tabla I}

\section{Clasificación de Woodard ${ }^{4}$}

\begin{tabular}{|l|l|}
\hline Categoría & Características \\
\hline \multirow{3}{*}{ I (20\%) } & Displasia renal \\
& Oligohidramnios \\
& Hipoplasia pulmonar y/o neumotórax \\
& Atresia uretral, uraco persistente \\
& Fascies de Potter \\
\hline \multirow{2}{*}{ II (40\%) } & Triada característica \\
& Displasia renal mínima o unilateral \\
& Ausencia de hipoplasia pulmonar \\
& Riesgo de urosepsis y/o azoemia \\
\hline \multirow{3}{*}{ III (40\%) } & Triada característica incompleta \\
& Uropatía moderada \\
& Ausencia de displasia renal \\
& Función renal normal \\
\hline
\end{tabular}

El síndrome también se encuentra re- y la malrotación de las asas intestinales ${ }^{4}$ lacionado con otras malformaciones. Las (tabla 2). más frecuentes son la hipoplasia pulmonar

\section{Tabla 2}

\section{Anomalías asociadas al síndrome de Prune-Belly ${ }^{4}$}

\begin{tabular}{|llll|}
\hline Pulmonar (58\%) & Cardiovascular (25\%) & Gastrointestinal (24\%) & Músculo esquelético (23\%) \\
\hline Hipoplasia pulmonar & Persistencia del conducto arterioso & Malrotación (40\%) & Escoliosis \\
\hline & Tetralogía de Fallot & Atresia vesical y de colon & Luxación congénita de cadera \\
\hline Defecto septal auricular & Torsión esplénica & Pie equinovaro \\
\hline Defecto septal ventricular & Ano imperforado & Pectus carinatum \\
\hline Anomalías valvulares & Gastrosquisis & Polidactilia \\
\hline & Onfalocele & Artrogriposis \\
\hline & $\begin{array}{l}\text { Anomalías en la división } \\
\text { de la cloaca }\end{array}$ & Hemivértebras \\
\hline & & Costillas cervicales bilaterales \\
\hline
\end{tabular}

Es ideal que el diagnóstico se realice de do fetal entre la $20^{\mathrm{a}}$ y $30^{\mathrm{a}}$ semana de gesmanera oportuna a través de un ultrasoni- tación. 
El diagnóstico posnatal se sospecha por clínica, al observar el abdomen en forma de ciruela pasa, la ausencia de testículos en el escroto y la palpación de otras vísceras a través del abdomen.

El tratamiento prenatal se lleva a cabo en casos de uropatía obstructiva severa; en estos casos, se realiza una derivación vésico-amniótica con el fin de prevenir la hipoplasia pulmonar. El tratamiento posnatal es quirúrgico, considerándose prioritario la orquidopexia y el abordaje del tracto urinario con propósito de la conservación y mejoría de la función renal.

Con respecto a la abdominoplastía, ésta no puede ser clasificada simplemente como una cirugía estética ${ }^{2}$ debido a que se ha demostrado que mejora el vaciamiento vesical.

Cada tratamiento debe individualizarse de acuerdo con los requerimientos y necesidades de cada paciente dependiendo del cuadro clínico, y determinarse como parte del progreso y mejoría de estos.

\section{Presentación del caso clínico}

Producto de madre de 28 años, gesta dos, la cual acudió a control prenatal en nueve ocasiones. Recibió suplementos como ácido fólico, hierro y calcio desde el primer trimestre del embarazo. Se realizaron siete ultrasonidos gestacionales, los cuales se reportaron anormales debido a la presencia de mega vejiga. Acude a consulta de urgencia y se detecta elevación de la presión arterial, leve edema de miembros superiores y proteinuria positiva, y se le diagnostica preeclampsia; una vez estabilizada la paciente, se decide realizar cesárea.

Se obtiene producto único, vivo, de 34 semanas de gestación, sexo masculino, $2000 \mathrm{gr}$ de peso, $40 \mathrm{~cm}$ de talla, sin datos de sufrimiento fetal.

El producto presenta apnea secundaria al nacimiento, por lo que se realizan maniobras avanzadas de RCP neonatal; se aspira, se intuba con cánula endotraqueal del número 3, se administra ventilación con presión positiva, inicialmente con bolsa y oxígeno, posteriormente con ventilador, se verifica permeabilidad esofágica. Se le asignó un Apgar de 6 al minuto y 9 a los cinco minutos.

Se reporta ausencia de rectos abdominales, estrías en pared abdominal (abdomen de ciruela pasa) y criptorquidia bilateral, dando como probable diagnóstico un síndrome de Prune-Belly (figura 1).

El paciente ingresa al servicio de Cunero Patológico 1 (CUPA 1), se toma radiografía de tórax, donde se observa expansión pulmonar adecuada. Se descarta hipoplasia pulmonar (figura 2). Se le toman exámenes de laboratorio, de los cuales se muestran algunos valores importantes: hemoglobina: $18.1 \mathrm{~g} / \mathrm{dL}$, hematocrito $54 \%$, plaquetas $45000 \mu \mathrm{L}, 4390$ leucocitos/ $\mu \mathrm{L}$, neutrófilos $2590 / \mathrm{mm}^{3}$, hemotipo O+, bilirrubinas totales $2.7 \mathrm{mg} / \mathrm{dl}$ a expensas de indirecta, TGP: $26 \mathrm{U} / \mathrm{L}$, TGO $38 \mathrm{U} / \mathrm{L}$, fosfatasa alcalina $118 \mathrm{U} / \mathrm{L}, \mathrm{DHL} 927 \mathrm{U} / \mathrm{L}$; se reportan células inmaduras, plaquetopenia y leucopenia. Durante la evolución, se encontró una uresis de $6.2 \mathrm{ml} / \mathrm{kg} / \mathrm{h}$, con un balance negativo en $24 \mathrm{~h}$ menor de $330 \mathrm{ml}$, reportándose al día siguiente un balance negativo menor de $240 \mathrm{ml}$.

Al tercer día, se agregó atelectasia apical derecha con sospecha de fístula vesicocutánea. Los signos vitales permanecen dentro de lo normal; se solicita ultrasonografía abdominal, renal y de vías urinarias; además de una gasometría arterial la cual dio como resultado una acidosis respiratoria. 
La evolución del cuadro continuó presentando campos pulmonares con estertores gruesos bilaterales, polipnea, tiraje intercostal y disociación toracoabdomina. Se solicitó una radiografía pulmonar de control donde no se encontraron datos patológicos persistentes.

El paciente fue referido a un hospital de tercer nivel de atención para la realización de un estudio multidisciplinario con su respectivo tratamiento. Hasta el momento de la hospitalización, no se contaba con cariotipo.

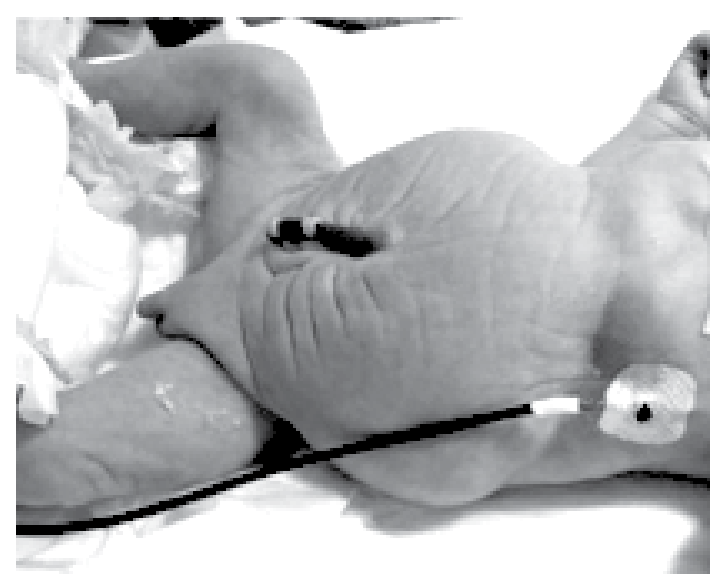

Fig 1

\section{Discusión}

El síndrome de Prune-Belly es una malformación congénita poco común en el mundo. Se calcula que afecta a 1 de cada 30,000 recién nacidos; es 20 veces más común en el sexo masculino que en el femenino. ${ }^{7,8}$

La etiología aún no se encuentra bien definida. Se cree que es debida a una anomalía mesodérmica, aunque en estudios recientes se ha encontrado una deleción del factor HNF-1ß, que se considera un factor necesario para la diferenciación visceral proveniente del endodermo. ${ }^{9-11}$

Algunos estudios reportan una correlación entre la edad de la madre (madres menores de 30 años) y la presencia del síndrome; sin embargo, aún no se encuentra algún factor materno predisponente. ${ }^{12}$

La enfermedad se caracteriza por una triada clásica: criptorquidia bilateral, au-

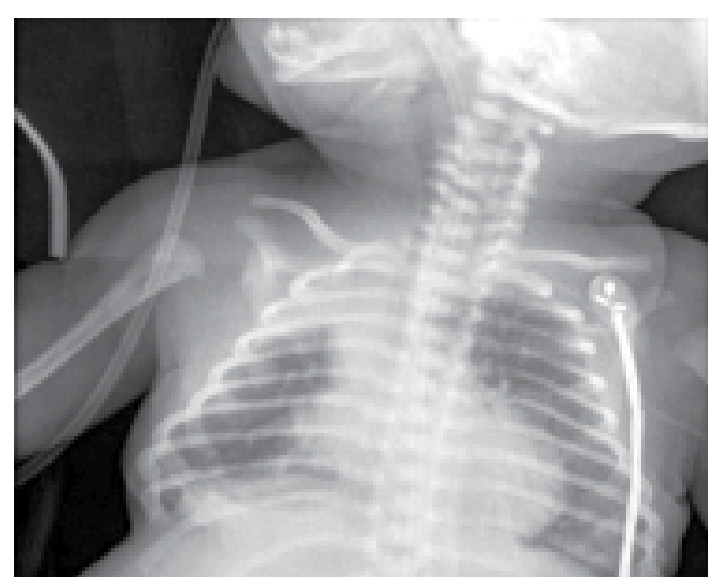

Fig 2

sencia de músculos de la pared abdominal y anomalías del tracto urinario; aunque puede estar acompañado de otras entidades patológicas como malformaciones pulmonares, musculoesqueléticas, cardiovasculares y gastrointestinales, como se reporta en la literatura. 3,4,13-15

El diagnóstico prenatal juega un rol importante en la detección temprana del síndrome. Se puede detectar a partir del segundo trimestre del embarazo a través de un ultrasonido, y los datos que orientan son: abdomen abultado, ausencia de pared abdominal, dilatación vesical, hidrouréter bilateral, hidronefrosis con parénquima hipoecoico o hiperecoico, así como oligohidramnios, ascitis fetal, pulmones hipoplásicos. $^{4}$

En nuestro medio, el diagnóstico suele hacerse de manera posnatal, sospechándose inicialmente por los datos clínicos des- 
de el momento del nacimiento; los datos esenciales son la ausencia de músculos abdominales y criptorquidia; también la asociación con el sexo masculino, aún pudiéndose encontrar casos en el sexo femenino. La mayoría de los pacientes son prematuros $y$, en caso de sospecha, se realiza un ultrasonido para detectar malformaciones urinarias.

Por ejemplo, en este caso se encontró la triada clásica del paciente, además de persistencia de uraco e hipofunción pulmonar al nacimiento. Estos fueron datos compatibles con el síndrome.

El pronóstico depende del compromiso de la función pulmonar y de la renal. La hipoplasia pulmonar es causa de la muerte en $30 \%$ a $50 \%$ de pacientes con síndrome de Prune Belly. ${ }^{15}$ La falla renal es la causa más común de complicación la cual conlleva al uso de terapia de reemplazo renal en algún momento de su vida.

El síndrome impacta de manera negativa en la calidad de vida de estos pacientes siendo importante el apoyo familiar y el tratamiento quirúrgico oportuno, como reporta Arlen y cols. ${ }^{1}$

Debido a lo antes mencionado, el diagnóstico debe realizarse de manera prenatal para brindar un tratamiento oportuno $y$, así, mejorar el pronóstico de los pacientes; para esto, es necesario que se lleve un control ultrasonográfico adecuado, con médicos especializados. Si el diagnóstico no se realiza en el control prenatal, éste se puede sospechar desde el momento del nacimiento, tal como sucedió en el caso mencionado; al sospecharse, es importante que se realicen todos los exámenes complementarios necesarios para confirmar el diagnóstico, tales como exámenes de laboratorio, ultrasonido de vías urinarias y radiografías de tórax, entre otros.

Finalmente, es importante que los médicos de pregrado y de primer contacto conozcan el síndrome pues, como se mencionó antes, el diagnóstico se puede realizar oportunamente desde el control prenatal o en su defecto, al momento del nacimiento, lo cual permite un manejo oportuno de la patología y evita complicaciones posteriores.

\section{Conclusiones}

El síndrome de Prune-Belly es una anomalía congénita con baja incidencia; sin embargo, es importante conocer sus características clínicas de esta entidad. Aún no se ha determinado algún factor etiológico que predisponga al desarrollo de la patología.

El diagnóstico se debe realizar de manera oportuna para brindar los tratamientos adecuados y prevenir las complicaciones que repercutan de manera negativa en la calidad de vida del paciente.

\section{Bibliografía}

1. Arlen, Angela M.; Kirsch, Susan S.; Seidel, Natan E.; Garcia-Roig, Michael; Smith, Edwin A.; Kirsch, Andrew J. Health-related Quality of Life in Children With Prune-belly Syndrome and Their Caregivers. Urology. 2015;87: 224-227.

2. Routh, Jonathan C.; Huang, Lin; Retik, Alan B.; Nelson, Caleb P. Contemporary Epidemiology and Characterization of Newborn Males with Prune Belly
Syndrome. Urology. 2010, 76(1). 44-48.

3. Samal SK, Rathod S. Prune Belly syndrome: A rare case report. J Nat Sc Biol Med. 2015;6:255-257

4. Hassett, S.; Smith, G.H.H.; Holland, A. J. A. Prune belly syndrome. Pediatr Surg Int. 2012, 28: 219-228.

5. Paul J. Murray, Katie Thomas, Christopher J. Mulgrew, Sian Ellard, Emma L. Edghill, Coralie Bingham. Whole gene deletion of the hepatocyte nuclear factor-1? gene in a patient with the prune-belly syndrome. NDT. 2008; 23(7): 2412-2415. 
6. Seidel, Natan E.; Arlen, Angela M.; Smith, Edwin A.; Kirsch, Andrew J. Clinical Manifestations and Managment of Prune-bely Syndrome in a Large Contemporary Pediatric Population. Urology. 2015; 85(1):211-215.

7. Tagore, Koyye Ravindranath; Ramineni, Asok Kumar S.; Lakshmi A.R.

8. M Kheir AE, M Kheir AE, A Ali EM, Medani SA, Maaty HS. Prune belly syndrome: A report of 15 cases from Sudan. Sudan J Paed. 2017; 17(1):42-48.

9. Mata-García, Luis Enrique; Chávez-Ocaña, Sonia. Síndrome de Prune Belly: revisión de la literatura a propósito de un caso. Rev Hosp Jua Mex. 2013; 80(2): 134-137.

10. Woolf, A.S., Stuart, H.M. \& Newman, W.G. Pediatr Nephrol (2014) 29: 353. doi:10.1007/s00467-0132472-1
11. Zugor, V., Schott, G. E., \& Labanaris, A. P. (2012). The Prune Belly syndrome: urological aspects and long-term outcomes of a rare disease. Pediatric Reports, 4(2), 78-81. doi:10.4081/pr.2012.e20

12. Ekwunife $\mathrm{OH}$, Ugwu JO, Modekwe V. Prune belly syndrome: Early management outcome of nine consecutive cases. Niger J Clin Pract 2014;17:425-30.

13. León-Hernández, M. A., Acosta-León, J., \& Cárdenas-Camarena, L. (2015). Síndrome de Prune Belly: cirugía para mejoría estética y reconstrucción abdominal. Cirugía Plástica Ibero-Latinoamericana, 41(2):175-178.

14. Hoyos $A$, et al. Sindrome de prune belly (ciruela pasa). MEDICINA UPB 2013; 29(2):155-161

15. Cabanillas $P$, et al. Síndrome de Prune Belly. Rev Chil Pediatria 2001; 72(2):135-138. 